Historic, Archive Document

Do not assume content reflects current scientific knowledge, policies, or practices. 



\title{
Gladiolus Bulbs and Bulblets Retail List Fall 1930
}

\section{MISS FLORA E. BRECK, Portland, Oregon}

\author{
384 E. 42 2nd St., $N$. \\ Ony stock has been inspected under Idicense Yo. 39s \\ L $1 \mathrm{H}-\mathrm{E} \& \mathrm{R}$

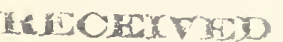 \\ NOV 61930 \&

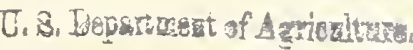

\section{IDISCOUNTS} \\ On all cash orders you may select extra Bulbs and Bulbleťs as follows: \\ $20 \%$ extra on orders from ..... \$50.00 to $\$ 100.00$ \\ $25 \%$ extra on orders from ..... 100.00 or nver
}

$5 \%$ extra on orders from ..... $\$ 5.00$ to $\$ 10.00$

$10 \%$ extra on orders from ..... 10.00 to 25.00

$15 \%$ extra on orders from ..... 25.00 to 50.00

\section{Safe Delivery Guaranteed by Express}

We guarantee safe delivery by express on orders of $\$ 5.00$ or more, if you notify us and your express agent at once in case of damage by freezing or other loss or damage, so that we may file claim.

\section{TERMS}

Cash or $25 \%$ down and balance C. O. D. All orders are received surject to prior sale. Discounts do not apply on spccial quotations. 1 at 9 th of 10 price. 10 at 9 times price per 1.

We pay transportation on orders over $\$ 3.00$

CI.OSIEI) ON SUND.MS

$$
\text { क्रा }
$$

\section{List of Vamoieties}

\section{GLADIOLUS}

A. E. Kunderd ( $K$, large ruffled salmon-rose Aida ( $K$ \& $M$ ) deep purple blue

Albatros (Pf.) extra fine tall pure white

Allen V. Bunce (Bill) orange, deeper throat stripes

Aloue (Par) soft salmon "Glad with pockets and spurs" Alton ( $\mathrm{K}$ ) beautiful ruffled orange

Alvina (E) exruisite flesh pink

Amethyst (Australia) excellent lilac rose

Ingel's Irean (E) fine La France pink

Imuie Laurie (Elown) shell pink. Florists like it

Apricot (Mair) salmon, many open

Apricot Glow (Can.) beautiful apricot

Are Maria (Pf.) excellent violet blue

Balboa ( $\mathrm{Br}$.) apricot pink

Beatricia (E) attractive red

Beatriz Micluelena (D) peach red

Beauty Wave (K) ruffled yellow, tinted pink

Beothoven (D) red

Belinde (Pf.) large carly sulphur yellow

Bengal Beauty (Mrs. E.) striking brown and gold ..........

Bengal Tiger (Pr.) brilliant mottled red

Besnard Shaw (D) lilac tinted white

Berty Snow (Mair) excellent lavender

Betty Joy (Bill) soft exeam white flushed pink

Betty Nuthall (Sal.) extra fine tall coral orange

Bill Sowden (Australia) very large deep red

Bird of Paradise (Crites) gorgcous cerise pink, large.

Black Pansy (K) rich dark red

Blenn (Bales) ruffled orange

Bobby (K) rose with deep blotches

Bobby Douglas (Rad.) beautiful creamy white

Break o'Day (Bill) very early light pink

Bronze Maid (E) deep bronze, purplish blue throat

Byorn I. Smith (K) popular lavender

\begin{tabular}{|c|c|c|c|c|}
\hline \multirow{2}{*}{\multicolumn{2}{|c|}{ Per No. 1}} & \multirow{3}{*}{$\begin{array}{c}\text { No. } 2 \\
11 / 4-11 / 2\end{array}$} & \multicolumn{2}{|c|}{ BULBS } \\
\hline & & & No. 3 & No. \\
\hline & $11 / 2$ up & & $1-11 / 4$ & $3 / 4-$ \\
\hline 10 & $\$ 1.75$ & $\$ 1.50$ & $\$ 1.25$ & $\$ 1.0$ \\
\hline 1 & .50 & .45 & .40 & \\
\hline 1 & .60 & .55 & .50 & \\
\hline 10 & ...... & ….. & ...... & 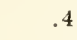 \\
\hline 1 & 100.00 & 100.00 & 100.00 & 100.0 \\
\hline 10 & .50 & .45 & .40 & 3 \\
\hline 1 & 1.00 & .80 & .60 & \\
\hline 1 & 1.00 & .75 & .60 & \\
\hline 1 & 1.00 & .85 & .65 & . \\
\hline 10 & 1.00 & .80 & .65 & \\
\hline 1 & 1.25 & 1.00 & .75 & \\
\hline 10 & 1.40 & 1.20 & 1.00 & .7 \\
\hline 1 & 1.75 & 1.50 & 1.25 & 1.0 \\
\hline 10 & .90 & .80 & .70 & .0 \\
\hline 10 & .45 & .40 & .30 & - \\
\hline 10 & .75 & .65 & .60 & \\
\hline 1 & .50 & .40 & .35 & \\
\hline 1 & .50 & .40 & .35 & \\
\hline 10 & 2.50 & $2.2 \overline{5}$ & 2.00 & 1.7 \\
\hline 1 & .75 & .65 & .55 & \\
\hline 10 & .45 & .40 & .30 & 2 \\
\hline 10 & 1.00 & .85 & .70 & 5 \\
\hline 10 & 2.00 & 1.70 & 1.35 & 1. \\
\hline 10 & 1.00 & .80 & .70 & \\
\hline 10 & 4.00 & 3.50 & 3.00 & \\
\hline 1 & 5.00 & 4.50 & 4.00 & 3.5 \\
\hline 1 & 5.00 & 4.50 & 4.00 & 3.5 \\
\hline 10 & ...... & $\ldots . .$. & ...... & 1. \\
\hline 1 & 5.00 & 4.50 & 4.00 & 3 . \\
\hline 10 & .65 & .55 & .45 & \\
\hline 1 & .75 & .70 & .65 & \\
\hline 10 & .45 & .40 & .30 & \\
\hline 1 & 2.50 & 2.10 & 1.70 & 1.3 \\
\hline 10 & .5 .5 & .45 & .35 & \\
\hline
\end{tabular}

BULELETS No. 5 No 6 Pel Price $1 / 2-3 / 4 \quad 1 / 2$ less $\$ .75 \quad \$ .50 \quad 100 \quad \$ .75$ $.30 \quad .25$

35 .30

00100.00

.20

$+40$

45

.35

40

.....

80

. 
Canbera (Frrey) pure lright yellow, heavy spike Capt. Courageous (E) large brilliant mottled red Cara Mia. (M) early pink

Cardinal Prince ( $K$ ) tall cardinal

Carmen Sylva (Dec.) popular white

Catlorine Colcuman (C) excellent tall deep pink

Clatice llowor ( $K$ ) tall ruffled creamy white

C'hicago (K) rutiled brick led

(Inicl Joseph (Pipel') tall eopper or brownish red

Col. Indberwl (H) red with white throat

Commodore (Mrs. E.) bright red, white throat

Coppor 13rolze (K) mottled copper bronze, fine

Coronado ( $\mathrm{Br}^{\circ}$ ) large white, purple throat

Coral Qnoen (Kernine) coral pink with yellow throat

('oryplice (Pf.) extra fine pink

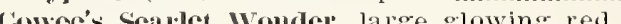

Creatore (Pill) deep salmon

('riusson Glow (Bet,) bright glowing crimson

$1 \frac{1 / 2}{\text { up }} 1_{1 / 4}^{1 / 1}-1 / 2$ 1-1 $1 / 4 \quad 3 / 4-1 \quad 1 / 2-3 / 4$ 1/2 less

Dicler's Mmerican Beanty, Ameriean beauty lose

Dr. Clist llaty (K) luffect led

I)r. I" Is Benucte (D) "House afire" red

Dr. Ifans Plitzel (Pf.) fine soft light pink

Dr. Moody (Kin.) large early lavender

Dorothy Wool (Mrs. E.) very early salmon

liarly Plapps (F) very early iridescent salmon

Eis.litl Wouder ( $K$ ) old rose, lar ce and smoky

lilizabet. Tabor' (H) early blotehed pink

WHell C. Jacoloscu (Mrs. E.) large tall pink

Elomil (E) deep pink

Vmile Aublun (L) rose, orange and sway

Thisma (K) a striking oddity

Finestine (Mrs, E.) orange, white throat

Cullolu (Fis.) amber yellow

Taitl (Kin.) lisht blue

lepu Kyle (K) ruffled creamy white

Nield Mawhal (Mrair) brownish red

Wrank M. Slick (Sal.) tall geranium pink

Fost King' (Mrs. E.) early and late coral pink

cicncul Canby (E) bright red, yellow feathered

Geo. J. Jocler (J) deep clear pink

Geraldime I'al'al' (D) lavender violet

Gertude Errey (Er, bright shell pint

Gladdle Boy (Bill) rose pink

Giadis I'latli (D) mallow purple

Alendale (Davis) tall purple

Gloriana (Bet.) extra fine salmon pink

Gloriola ( $\mathrm{E})$ peach red, yellow throat

Gold Iingle (A) early golden yellow

Golden Chimes (F) ruffled wide-open yellow

Golden Dreann (Gr.) tall deep yellow

Goldon Frllls (K) deep ruffled yellow

Golden Measure (Kel.) large tall yellow

Goldstome ( Hd.) large sparkling orange red

Groy In niglt (E) the Iris lavender

Ifalcyou (Jill) tall rose pink

Ifcavenly Blue (K.\& M.) tall lavender blue

IGeleu Wills (Sal.) tall irory white, cream throat

Iferuda (A) magenta

Ilerbstzamber (Pf.) late orange salmon

llishlaud Laddic ( $K$ ) mottled pink

llimenou ( 1 ustralia) very striking oldity, leep pink

Ilope Ilanpton (D) mottled strawberry pink ............... 10

II. C. IVolls (D) purplish red

Imperator (Hol.) large creamy white

Iniproved Ifenry ford (E) deep purple

Improved Oneen of the Niwht (D) rery dark red

.r. I. Carbonc (D) iridescent orange

back Iondon (D) orange salmon

- bacobir vall Beijeren (Eu.) tall purple

Joluy lind (Dec.) pink and cream

Jessic (Mair) deep pink

Joern's White (.I) very large white

Jolut Dover ( $\mathrm{K}$ ) deep lavender

Jolın T. Mrcuteheon (K) prolific red

Tonklicer van Tets (Pf.) fine pure white

Jubilee (Kemp) large lavende

onuiala (Kemp) pink with white throat

King George (Mair) large red, white throat

lipolage's Parorite (Kre.) one of the best red prims

líndrod's Vellow Wonder, vigorous yellow ....................... 10

lady Iolene (E) exquisite deep pink, yellow throat .......

Lady Machetl (Mrs, fo, red with white throat

Ladly l'rincra. (H) light color, fine for florists

Lady of the Make (E) fine pink

Iavender Bride, tall smoky lavender

Tilac Wondep ( $\mathrm{Eu}$.) lavender lilac

Lomgtellow (Dec.) tall slender pink

Jos Angeles (Hd.) "Cut and Come" pink

\begin{tabular}{|c|c|c|c|c|c|c|c|}
\hline 1.00 & .80 & .70 & & .45 & .30 & 10 & .55 \\
\hline 1.75 & 1.50 & 1.25 & 1.00 & .75 & .50 & 10 & .50 \\
\hline .50 & .40 & .30 & .25 & .20 & .15 & 10 & .25 \\
\hline 1.00 & .85 & .70 & .55 & .40 & .25 & 100 & .25 \\
\hline .45 & .40 & .30 & .25 & .20 & .15 & 100 & . 1 i \\
\hline .90 & .70 & .55 & .45 & .40 & .35 & 100 & .35 \\
\hline 1.00 & .85 & .70 & .55 & .40 & .25 & 100 & . \\
\hline .75 & .60 & .50 & ....... & .30 & .25 & 10 & .35 \\
\hline .50 & .45 & .40 & .35 & .30 & .25 & 10 & .25 \\
\hline 8.00 & 7.00 & 6.00 & 5.00 & 400 & 3.00 & 10 & .50 \\
\hline 1.00 & .90 & .80 & .70 & .50 & .40 & 10 & .40 \\
\hline .75 & .65 & .50 & .40 & .35 & .30 & 100 & .30 \\
\hline 1.00 & .90 & .80 & .70 & .60 & .50 & 10 & .75 \\
\hline .90 & .80 & .70 & .60 & .50 & .40 & 10 & .50 \\
\hline 1.00 & .90 & .80 & .70 & .60 & .50 & 10 & .75 \\
\hline .45 & .40 & .30 & .25 & .20 & .15 & 100 & .15 \\
\hline .50 & .4 .5 & .40 & .35 & .30 & .25 & 10 & .20 \\
\hline .45 & .40 & .30 & .25 & .20 & .15 & 100 & .15 \\
\hline .45 & .40 & .30 & .25 & .20 & .15 & 100 & .15 \\
\hline .75 & .65 & .55 & .45 & .35 & .25 & 100 & .20 \\
\hline .55 & .45 & .35 & .30 & .25 & .20 & 100 & .20 \\
\hline .45 & .40 & .35 & .30 & .25 & .20 & 10 & .20 \\
\hline .40 & .35 & .30 & .25 & .20 & .15 & 10 & .20 \\
\hline 2.25 & 2.00 & 1.75 & 1.50 & 1.25 & 1.00 & 100 & 1.25 \\
\hline 2.25 & 2.00 & 1.75 & 1.50 & 1.25 & 1.00 & 100 & 1.25 \\
\hline .50 & .45 & .40 & .35 & .30 & .25 & 10 & .30 \\
\hline .45 & .40 & .30 & .25 & .20 & .15 & 100 & .15 \\
\hline 1.00 & .90 & .80 & .70 & .60 & .50 & 10 & .75 \\
\hline 1.25 & 1.05 & .85 & .65 & .45 & .25 & 100 & .45 \\
\hline 1.50 & 1.25 & 1.00 & .75 & .50 & .25 & 100 & .50 \\
\hline 1.25 & 1.05 & .85 & .65 & .45 & .25 & 100 & .40 \\
\hline .75 & .65 & .55 & .45 & .35 & .25 & 10 & .35 \\
\hline .55 & .45 & .35 & .30 & .25 & .20 & 100 & .20 \\
\hline 10.00 & 8.00 & 5.00 & 4.00 & 3.00 & 2.50 & 10 & 9.00 \\
\hline .45 & .40 & .30 & .25 & .20 & .15 & 100 & .15 \\
\hline 2.00 & 1.75 & 1.50 & 1.25 & 1.00 & .75 & 10 & 2.00 \\
\hline 5.00 & 4.25 & 3.50 & 2.75 & 2.00 & 1.25 & 100 & 1.50 \\
\hline .80 & .70 & .60 & .50 & .40 & .30 & 100 & .35 \\
\hline 1.50 & 1.25 & 1.00 & .75 & .50 & .25 & 10 & .35 \\
\hline 1.50 & 1.25 & 1.00 & .75 & .50 & & 100 & .25 \\
\hline 1.25 & 1.05 & .85 & .65 & .45 & .25 & 100 & .30 \\
\hline .45 & .40 & .30 & .25 & .20 & .15 & 100 & .15 \\
\hline .65 & .55 & .45 & .35 & .30 & .25 & 100 & $.2 \overline{5}$ \\
\hline .45 & .40 & .30 & .25 & .20 & .15 & 100 & .15 \\
\hline .45 & .40 & .30 & .25 & .20 & .15 & 100 & .15 \\
\hline .90 & .70 & .55 & .45 & .40 & .35 & 100 & .35 \\
\hline 1.50 & 1.30 & 1.10 & .90 & .70 & .50 & 10 & .75 \\
\hline .65 & .5 .5 &.$\$ 5$ & .35 & .311 & .25 & 100 & .25 \\
\hline 20.00 & 17.50 & 15.00 & 12.50 & 10.00 & 750 & 1 & 2.00 \\
\hline 1.10 & . & .80 & .65 & .50 & .35 & 100 & .30 \\
\hline .85 & .70 & .55 & .40 & .25 & .15 & 100 & .20 \\
\hline .55 & .45 & .35 & .30 & .25 & .20 & 100 & .20 \\
\hline .75 & .60 & .50 & .40 & .30 & .25 & 10 & .50 \\
\hline 1.50 & 1.30 & 1.10 & .90 & .70 & .50 & 10 & .75 \\
\hline .85 & .70 & .55 & .40 & .25 & .15 & 100 & .20 \\
\hline 2.25 & 1.95 & 1.65 & 1.35 & 1.05 & .75 & & \\
\hline \multirow{2}{*}{.30} & .25 & .20 & & & .15 & 10 & .25 \\
\hline & & & .35 & .25 & .15 & 160 & .15 \\
\hline 1.25 & 1.05 & .85 & .055 & .45 & .25 & 100 & .30 \\
\hline & & & .30 & .25 & .20 & 100 & .20 \\
\hline 2.00 & 1.75 & 1.50 & 1.25 & 1.00 & .75 & $10^{\circ}$ & 1.00 \\
\hline & 1.25 & 1.00 & .85 & .70 & .55 & 100 & .75 \\
\hline & 1.25 & 1.00 & .85 & .60 & .35 & 100 & .50 \\
\hline 1.00 & .90 & .80 & .70 & .60 & .50 & 100 & .30 \\
\hline 10.00 & 8.75 & 7.50 & 6.25 & 5.00 & 3.75 & 1 & 1.00 \\
\hline .75 & .60 & .50 & .40 & .30 & .20 & 100 & .25 \\
\hline .45 & 40 & .30 & .25 & .20 & .15 & 100 & .15 \\
\hline .45 & .40 & .30 & .25 & .20 & .15 & 100 & .15 \\
\hline .55 & .45 & .35 & .30 & .25 & .20 & 100 & .20 \\
\hline $.4 \pi$ & .40 & .30 & .25 & .20 & .15 & 100 & .15 \\
\hline 1.25 & 1.15 & 1.00 & .80 & .60 & .40 & 10 & .50 \\
\hline 1.75 & 1.50 & 1.25 & 1.00 & .75 & .50 & 100 & .60 \\
\hline .90 & .75 & .65 & .50 & .35 & .25 & 100 & .25 \\
\hline & .75 & .65 & .50 & .35 & .20 & 100 & .20 \\
\hline .75 & .60 & .50 & .40 & .30 & .15 & 100 & .20 \\
\hline 2.50 & 2.00 & 1.50 & 1.25 & 1.00 & .75 & 10 & 1.00 \\
\hline 2.00 & 1.50 & 1.00 & .75 & .50 & .25 & 10 & .70 \\
\hline .90 & .70 & .55 & .45 & .40 & .35 & 100 & .35 \\
\hline .40 & .35 & .30 & .25 & .20 & .15 & 10 & .15 \\
\hline 1.20 & 1.00 & .80 & .60 & .50 & .40 & 100 & .35 \\
\hline .65 & .55 & .45 & .35 & .30 & .25 & 100 & .25 \\
\hline 1.50 & 1.30 & 1.10 & .90 & .70 & .50 & 10 & .75 \\
\hline 4.50 & 3.75 & 3.00 & 2.25 & 1.60 & 1.00 & 100 & 2.50 \\
\hline .75 & .65 & .55 & .45 & $\ldots \ldots$ & .35 & 10 & .40 \\
\hline .60 & .55 & .50 & .45 & .35 & .25 & 10 & .35 \\
\hline 3.50 & 3.00 & 2.50 & 2.00 & 1.50 & 1.25 & 100 & 1.25 \\
\hline .85 & .75 & .65 & .55 & .45 & .35 & 100 & .35 \\
\hline .55 & .45 & .35 & .30 & .25 & .20 & 100 & .20 \\
\hline & 40 & 30 & .25 & .20 & .15 & 100 & 5 \\
\hline
\end{tabular}


Madam Momet-Sully (Eu.) pure white, bricht red throat 10 llammoth White (U. B.) largest pure white, excellent... Marie Kunderd ( $K$ ) early white with pointed petals ....... 1 Marietta (MI) extra fine tall salmon

Maruori (Australia) Maruia (Kemp) grenadine orange

Maroceo (Pfi.) deep dark red

Marshal Foclı ( $K$ ) beautiful large salmon pink

Mary Frey (G) good deep lavende

Mary Lockwood (Eu.) tall pink

Mary Gardel (Piper) white with lavender markings

Mary o*Mine (B) very large pure white

Mary Pickford ( $K$ ) dainty white and cream

Masterpiece $(K)$ ruffled American beauty

Minnet (C) great prize-winning lavender

Iiss Canby (E) daintily ruffled creamy white

Miss Joy (Holmes) La France pink, light throat

Monarch of the Glen (Mrs. E.) heary tall ruffled pink

Mother Machree (St.) unusual tall smoky

Momntan Girl (MIrs. E.) florists' fine lavender pink

Mrs. Imma Pfitzer (Pf) greenish white, many open

Mrs. Calvin Coolidge ( $K$ ) pink with yellow throat

Irs. Dr. Norton (K) white tinted with pink

IIs. F. C. IIomberor (Hor.) white, popular

Mrs. F. C. Peter's (Fis.) tall lavender

Mrs. Galbraith (Mair) large salmon

Irs. I. F. Miller (MI) ruffled pink, yellow throat

Mrs. Leon Donglas (D) very tall salmon

Mrs. P. IV. Sisson (C) famous ruffled pink

Mrs. Robt. Commingham (D) tall lavender

Irs. T. IRattray (Australia) very tall rose pink

Mr. W. H. Phipps (D) famous iridescent pink

Ocelhs ( $\mathrm{B}$ ) lavender with deep violet blotch

Orange Flame (K) large vermillion orange

Orange Qneen (Hol.) orange

Orehid Lady (Sp.) large deep lavender

Oregon Qneen (Mrs. E.) large lily-like cream

Otlrello (Pf.) violet purple

On' Selection (Australia) purple-lavender salmon

Patrieia Carter (K) early light shrimp pink

Paul Pritzer (Pf.) brilliant velvety purple

pearl of California (King.) soft La France pink

Pfitzer's Trimmph, wonderful large orange red

Inilatelia (K) large gorreous rose

l'halloule ( $\mathrm{Eu}$ ) salmon pink, yellow theot

Phramia (Russel) tall orchid lavender

Pink Cloud (K) ruffled rose pink

P'ink Marie (E) fine florists' pinl

Pink Lily (K) soft purplish pink

Point 'Wwan (E) large pink with pointed petals

Pride of Wanakah (Chr.) lar'ge lavender rose

Pride of portland (E) large begonia-rose pink

Primrose (A ustralia) yellow

Princely (Kemp) fine florists' white

Prince of Wales ( $V$, Z.) very early salmon pinl

Princess Mary (MIair) cream shaded pink, many open

Pnrest of All (Pf.) pure white

Purple Glory (K) ruffled deep red

Pnrple Queen ( $\mathbf{K}^{*}$ ) deep purple

Queen of Brencen (Zim.) good pink prim.

Red Fire ( $K$ ) very red, stands heat well

Rod Glory (E) extra fine large ruffled red

Red Roy (E) fine red, tall slender spike

Red Tornado (E) very brilliant red, heavy texture

Richald Dienel" (D) famous pink

Rippling Waters (E) eream, tinted with pink

Rita Beck (Fis.) large pink

lRobt. J. Kunderd ( $K$ ) fine tall scarlet red

Rose Isl (D) vimorous ashes of roses

Rose of Califoruia. ( $\mathrm{Hd}$.) rose

Ruffled Gold (G) splendid bright yellow

Ruffolace (Kemp) lacinated white

Sacajawea (Pratt) tall coppery bronze

Salbach's Pink (Salb.) large deep pink, extra good

Santa Barbara ( $\mathrm{Br}$ ) fine lavender tinted cream white

Saraband (Salb.) lich velvety borteaux

Senrlet Bedder (Salb.) glowing scarlet

Serrita (Bill) lacinated cream

Souneuber. (Bill) laro creamy pink

Spirit of St. Couis (D) buff tinted orange red

Star of the Sea ( $E)$ peach red, brownish throat

smmymede (Fis.) light amber orange

Sumsliue Girl (Sterens) soft salmon, clear throat

Sword of Malnouet (E) oriental red, purplish smok

Sylvia Wost (Rug.) small pure white, many open

Tamalpais (D) tall orange red

The Orchid (Spr.) lacinated lavender pink

Thos. A. Edison (1) begonia rose

Thos. A. Ealison ( $K$ ) very dark ruffled red

Tyran Beanty (K) American Peauty lose

No. 1 No. 2 No.3 No.

$11 / 2$ up $11 / 4-11 / 2 \quad 1-1 \frac{1 / 4}{3} \quad 3-1$

\begin{tabular}{|c|c|}
\hline 2.00 & 1.75 \\
\hline 1.25 & 1.00 \\
\hline 45 & .40 \\
\hline .55 & .45 \\
\hline .45 & .40 \\
\hline .45 & .40 \\
\hline 1.50 & 1.25 \\
\hline .50 & $4 i$ \\
\hline .40 & .35 \\
\hline 1.00 & .811 \\
\hline 1.00 & .90 \\
\hline .40 & (35) \\
\hline .45 & .40 \\
\hline 15 & .40 \\
\hline 1.50 & 1.25 \\
\hline.+0 & 35. \\
\hline . +0 & .35 \\
\hline 3.00 & 2.50 \\
\hline 5.00 & 4.50 \\
\hline .50 & .45 \\
\hline 3.00 & 2.50 \\
\hline 2.25 & 1.85 \\
\hline .45 & .40 \\
\hline 1.00 & .85 \\
\hline .45 & .40 \\
\hline 1.75 & 1.50 \\
\hline 25.00 & 20.00 \\
\hline .45 & .40 \\
\hline .65 & .55 \\
\hline 1.25 & 1.10 \\
\hline 1.00 & .60 \\
\hline .65 & $.5 \overline{3}$ \\
\hline .75 & .60 \\
\hline 1.00 & .90 \\
\hline
\end{tabular}

1.25

1.00

.5

.20 
Chlan Chief $(\mathrm{K})$ tall red

Veilchenblau (Pf.) violet blue, deeper throat

Veiled 13rilliance (A) large gray tinted pink

Victor (Australia) red, white throat brilliant contrast...

W. A. Sisson (D) lal.ge light lavender pink

White Cloud (E) tall white, many open

Villiam Doneaster (N, Z . )

Winged Vietory ( $\mathrm{Br}$.) large airplane pink

Wotan (D) bright pink with blotch

W'm. Kent (D) rich ruffled cream

Yellow Perfection (Pf.) fine pure soft yellow

$\begin{array}{rrrr}.75 & .65 & .55 & .45 \\ .40 & .35 & .30 & .25 \\ 2.00 & 1.60 & 1.20 & .80 \\ 3.50 & 3.00 & 2.50 & 2.00 \\ 1.10 & .95 & .80 & .65 \\ .40 & .35 & .30 & \ldots \\ .50 & .45 & .40 & .35 \\ 1.00 & .90 & .80 & .70 \\ \ldots \ldots & . . .5 & 1.25 & 1.00 \\ .85 & .75 & .65 & .55 \\ 1.25 & 1.00 & .75 & .60\end{array}$

$1 / 2-3 / 4 \quad 1 / 2$ less

$\begin{array}{rrrr}.35 & .25 & 100 & .25 \\ .20 & .15 & 10 & .20 \\ .60 & .40 & 100 & .30 \\ 1.50 & 1.25 & 100 & 2.00 \\ .40 & .20 & 100 & .40 \\ \ldots \ldots & .15 & 10 & .20 \\ .30 & .25 & 10 & .20 \\ \ldots \ldots & . . .5 & 10 & 1.00 \\ .80 & .65 & 100 & 1.00 \\ .40 & .25 & 100 & .30 \\ .50 & .35 & 10 & .75\end{array}$

\section{"xis}

\section{Special Glad Diferes}

We have a number of varieties in too small quantities to list regularly but can sparc bulblets of the following varieties in fairly small amounts. Therefore-

You May Select 200 Bulblets of the Following Varieties for $\$ 5.00$

subject to the following requests:

Of the starred varieties, do not ask for more than five bulblets of any one variety.

Do not select more than 15 of the starred varieties in one $\$ 5.00$ order.

Do not ask for less than 15 nor more than 25 bulblets of any un-starred variety.

You may order more than one $\$ 5.00$ set of bulblets if you like.

If possible, please suggest one or two substitute varieties from among this list in case we are out of some of those you choose. Or, would you prefer to have as a possible substitute a blooming size bulb of one of the following: Commodore, Angel's Dream, Betty Nuthall, Bengal Beauty, Gay russar, or Pride of Portland? If so, plcase state your preference.

Aflame ( Hornberger)

*Antione ( $\mathrm{K}$ )

Alma Rubens (D)

Admiral Beatty (Vil.)

Barhara La Marr (D)

* Bridal Veil (A)

* Betty Lucile (Kin.)

* Borrowed Gold

Buckeye (K)

Buddy Worden

Crinkles ( $\mathrm{K}$ )

Capt. Jinks

Chas. Dickens (Pf.

California (Kir.)

* Chas. Berthier (Vil.)

* Crown of Gold (Bill)

Cleopatra (Bill)

D'Aguesseau (France)

Dignity (Mair)

Dr. Elkins ( $\mathrm{K}$ )

* Duchess of Atholl (Mair)

Duchess of York (Mair)

* Dawn of Peace (H)

Dr, Nelson Shook (K)

Tilegance (Mair)

Flaming Sword (Hol.)

Fay Lanphier (Williams)
Fuld's Favorite (K)
Frilled Felicity (Kir.)
Goldquille
George Vits
Golden Orange (K)
Glenburn (Mair)
Gold Star
Tranhoe (Australia)
Iwa (Betscher)
*Ivernia (Errey)
Jap Lady
*Joerg's Yellow Prim.
James Dunlop (Mair)
* Kyle (Mair)
* Krimhilde (Pf.)
"Little John (Stcvens)
*Lillian Gish (D)
Le Cygne (Hd.)
*Linalee (D)
Lady Alice (Barber)
*Louvois (France)
(Mauve rose, very good.)
Laverna (Errey)
Laidley (Eirey)
Lady Esther (C)

Le Roi Lear (Vil.)
Madam Geo.Boulay (Lem.)
Miss Demure (A)
Mongolia (K)
Matarika (N. Z.)
Melissa (Errey)
Mrs. Beckwith
Marvel (Mair.)
* May MacAvoy (D)
Milton Sills (D)
Mrs. Van Konynenburg (Pf.)
Mary Jane (K)
Molly Douglas (N. Z.)
*Mabel (Mair)
Mrs. A. B. C. Dohrman (D)
*Mary Van (Kin.)
*Mrs. S. A. Errey (Errey)
*Mrs. Dr. F. E. Bennett
Mrs. Gertrude Pfitzer (Pf.)
*New Violet (Kir.)
Nixie (D)
Oh Boy (Bill)
*Oraflame (Kemp.)
*Orange Wonder (Kemp.)
"Othahuhu (N. Z.)
*Orange Butterfly (Sal.)

Peach Rose (K)

* Prof. Van Slogteren (Alk.) * Primrose Princess (Sal.) Pride of Oregon (Damon) Peach Amber (Miller)

* Purple Victory (Gilrey)

* Pres, Hoover (Kin.) Persia (Gen. Bulb)

Roodkapje (Hol.)

* Rosewood (A)

Red Orange ( $\mathrm{K}$ ) Ruby (Kir.)

Rich and Rare ((Kelway) * Resplendent

* Roi Albert (Lemoine)

Remus (Australia)

Romance (K)

Rosalind ( $\mathrm{K}$ )

*Te Whiti (N. Z.)

Troubadour (Pf.)

*Vesuvius Glow

Van (Stevens)

Wonderful (K)

Wedgewood

Yellow Wonder ( $\mathrm{Hol}$ )

NOTE: Perhaps you may prefer to have Bulbs of some of the above varieties. If so, MAKE A SET YOURSELF

stating the number and sizes you wish and if we can furnish it we will be glad to make you a quotation.

Substitution: If out of the size ordered, we will substitute as near as possible to the size ordered in the same variety. If entirely out of that variety, shall we substitute with more than equal value of a variety that is in our opinion, a better variety, applying the golden l*ule in substitutions?

Please do not order less than 20 cents worth of any item.

TWere you ever disappointed in expensive bullolets failing to gcrminate? We want our customers satisfied. Therefore, with every order, if requested, we will enclose instructions for germinating bulblets, If these instructions are followed calefully, we believe you will get a high percentage of rernuination.

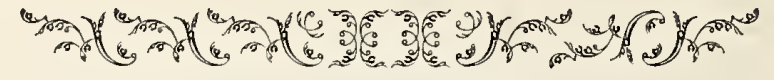

\section{Various Seed Offers}

Oregon Giant Pansies, 600 seeds ........\$ $\$ 1.00$ Regal Iily seed, per pound ............. 6.00 Wrexham Delphinium seed, per plet. ...... 1.00 Columbine seed, per pkt. 300 seeds ....... $\quad .50$ Double Poppies, seed, per plit. .......... .15

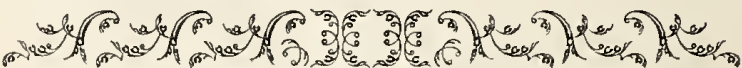

Please notice that our Retail List is priced Prepaid but the Wholesale List is priced F. O. B. 